\title{
Correction to: Homological multiplicities in representation theory of $p$-adic groups
}

\author{
Avraham Aizenbud $^{1}$. Eitan Sayag ${ }^{2}$ \\ Published online: 17 July 2021 \\ (c) Springer-Verlag GmbH Germany, part of Springer Nature 2021
}

\section{Correction to: Mathematische Zeitschrift (2020) 294:451-469 https://doi.org/10.1007/s00209-019-02262-4}

\section{Correction of $[1, \S 6]$}

Regrettably, [1, Lemma 6.4] is incorrect, even though the statements of the theorems in [1] are correct with no modification required. However, [1, Theorem 6.1], whose proof relied upon the wrong [1, Lemma 6.4], requires a different proof which we provide here. We note that the conclusion of [1, Lemma 6.4] holds true for the case of symmetric pairs and, more generally, in the factorizable case (see Chapter 9 of [4]). The rest of [1] requires no modification.

A corrected version of the original paper [1] will appear on the Arxiv.

For the proof of Theorem [1, Theorem 6.1] we require a few preparations. We employ the notation of [1] and, specifically, those of $[1, \S 6]$.

Lemma 1.1 Let a discrete countable group $\Gamma$ act freely on a locally compact space $Y$. Assume that $\Gamma \backslash Y$ is Hausdorff. Let $\phi: Y \rightarrow \Gamma \backslash Y$ be the quotient map. Then there is an open nonempty set $U \subset \Gamma \backslash Y$ such that $\phi^{-1}(U) \cong \Gamma \times U$ as a $\Gamma$-space.

Proof Let $E=\{(x, y) \in Y \times Y \mid \exists g \in \Gamma$ such that $y=g x\}$ be the equivalence relation induced by the action $a: \Gamma \times Y \rightarrow Y$. By the assumption $E$ is closed in $Y \times Y$, hence locally compact. We have $\Gamma \cdot \Delta Y=E$, thus, by Baire's category theorem, there is a $g \in \Gamma$ such that $g \Delta Y$ is not nowhere dense in $E$, therefore neither is $\Delta Y$ nowhere dense in $E$. Since $\Delta Y$ is closed in $Y \times Y$, it is also closed in $E$. Consequently, the interior of $\Delta Y$ in $E$ is not empty, thus we have a non-empty open set $V \subset Y$ such that $\Delta V$ is open in $E$. As a result, we have

The original article can be found online at https://doi.org/10.1007/s00209-019-02262-4.

$\triangle$ Eitan Sayag

sayage@math.bgu.ac.il

http://www.math.bgu.ac.il/ sayage/

Avraham Aizenbud

aizenr@gmail.com

http://www.wisdom.weizmann.ac.il/ aizenr/

1 Faculty of Mathematical Sciences, Weizmann Institute of Science, 76100 Rehovot, Israel

2 Department of Mathematics, Ben-Gurion University of the Negev, Be'er Sheva, Israel 
some open $W \subset Y \times Y$ such that $W \cap E=\Delta V$. Let $x \in \Delta V$. Let $A, B \subset Y$ be open sets such that $x \in A \times B \subset W$. Let $C:=A \cap B$. We then get $x \in C \times C \subset W$ as well as $C \times C \cap E=\Delta C$. Let $U:=\phi(C)$. The map $\left.\phi\right|_{C}: C \rightarrow U$ is an open bijection, thus a homomeorphism. Let $\psi$ be its inverse. Consider the composition

$$
\Gamma \times U \stackrel{i d \times \psi}{\rightarrow} \Gamma \times C \stackrel{\left.a\right|_{\Gamma \times C}}{\rightarrow} \phi^{-1}(U) .
$$

This is a $\Gamma$-equivariant open surjection. Since the action of $\Gamma$ of $Y$ is free this composition is also injective. Thus it is bijective, and gives the required homeomorphism.

Corollary 1.2 Let $S$ be a locally compact group. Let $Y$ be a transitive locally compact $S$ space. Let a discrete countable group $\Gamma$ act freely on $Y$, commuting with the action of $S$. Assume that $\Gamma \backslash Y$ is Hausdorff. Then:

1. The map $\phi: Y \rightarrow \Gamma \backslash Y$ is a locally trivial fiber bundle.

2. If, in addition, $Y$ is totally disconnected and countable at infinity, then $\phi$ is a trivial fiber bundle.

Proof 1. Since the action of $\Gamma$ commutes with the action of $S$ on $Y$, the latter descends to an action on $\Gamma \backslash Y$. By Lemma 1.1 we have a non-empty $U \subset \Gamma \backslash Y$ such that $\phi^{-1}(U) \cong \Gamma \times U$ as a $\Gamma$-space. Thus for any $g \in S$, we have $\phi^{-1}(g U) \cong \Gamma \times g U$ as a $\Gamma$-space. This implies the local triviality of $\phi$, since the action of $S$ on $\Gamma \backslash Y$ is transitive.

2. The space $Y$ has a countable basis consisting of open compact sets. Their images in the quotient $\Gamma \backslash Y$ form a countable basis for the topology of $\Gamma \backslash Y$ consisting of open compact sets. Therefore any open cover of $\Gamma \backslash Y$ has a refinement which is disjoint. This, together with the previous item, proves the assertion.

Corollary 1.3 Let $S$ be an l-group and let $Y$ be an l-space with a transitive action of S. Let $\Gamma$ be a discrete countable group acting freely on $Y$ and commuting with the action of $S$. Assume that $Y$ is countable at infinity and that $\Gamma \backslash Y$ is Hausdorff. Let $\mathcal{L}$ be an $\Gamma$-equivariant sheaf on $Y$. Then $\mathcal{S}(Y, \mathcal{L})$ is a free $\mathcal{H}(\Gamma)$-module.

Proof By previous corollary (Corollary 1.2(2)), $Y \cong \Gamma \backslash Y \times \Gamma$ as a $\Gamma$ space. Thus there is a vector bundle $\mathcal{L}^{\prime}$ on $\Gamma \backslash Y$ such that $\mathcal{L} \cong \phi^{*}\left(\mathcal{L}^{\prime}\right)$, where $\phi: Y \rightarrow \Gamma \backslash Y$ is the quotient map. This implies that $\mathcal{S}(Y, \mathcal{L}) \cong \mathcal{H}(\Gamma) \otimes \mathcal{S}\left(\Gamma \backslash Y, \mathcal{L}^{\prime}\right)$.

Lemma 1.4 Let $\phi: S_{1} \rightarrow S_{2}$ be a surjective continuous homomorphism ofl-groups. Assume that $S_{1}$ is countable at infinity. Then $\phi$ is open.

Proof It is enough to show that, for any compact open subgroup $K<S_{1}$, the group $\phi(K)$ is open. Note that $S_{1} / K$ is countable. We get $S_{2}=\bigcup_{i} h_{i} \phi(K)$ for some sequence $\left(h_{i}\right) \subset S_{2}$. By Baire's category theorem we get $i$ such that $h_{i} \phi(K)$ has non-empty interior. Hence, $\phi(K)$ has non-empty interior. Since $\phi(K)$ is a group, the fact that $\phi(K)$ has non-empty interior implies that it is open.

To guarantee the Hausdorff property we will use:

Lemma 1.5 Let $\Lambda_{\mathcal{Z}}$ be as in [1, Lemma 6.3]. Let $\Gamma<\Lambda_{\mathcal{Z}}, \mathbf{H}<\mathbf{G}$ and put $H:=\mathbf{H}(F)$. Assume that $\Gamma \cap H$ is trivial. Then $H \Gamma \subset G$ is closed.

Proof Let $\mathbf{T}:=\Gamma \otimes_{\mathbb{Z}} \mathbb{G}_{m}$. We have a natural homomorphism of algebraic groups $\psi: \mathbf{T} \rightarrow$ $Z(\mathbf{G})$. It gives rise to a homomorphism $\alpha: \mathbf{H} \times \mathbf{T} \rightarrow \mathbf{G}$. We will now show that $\alpha$ has 
a finite kernel. Let $\mathbf{K}$ be its kernel and $\mathbf{K}^{\prime}$ be its projection to $\mathbf{T}$. If $\mathbf{K}$ is infinite, then so is $\mathbf{K}^{\prime}$ and hence $\mathbf{K}^{\prime}$ has a split non-trivial subtorus $\mathbf{T}^{\prime}$. Let $m: \mathbb{G}_{m} \rightarrow \mathbf{T}^{\prime}$ be a nontrivial homomorphism. Such a homomorphism gives a non-trivial element in $\Gamma \cap H$, contradicting our assumption. We thereby conclude that $\alpha$ has a finite kernel.

Let $Q:=\operatorname{Im}\left(\alpha_{F}\right)$ where $\alpha_{F}: H \times T \rightarrow G$ is the map induced by $\alpha$ on $F$-points. By [3, Theorem A.2], $Q$ is constructible. Since $\alpha_{F}(T)$ is central, $Q$ is a subgroup, and is therefore closed. By Lemma 1.4 the map $\alpha_{F}$ when considered as a map $H \times T \rightarrow Q$ is open. Therefore it remains to be shown that $\alpha_{F}^{-1}(H \Gamma)$ is closed. This follows from the finitness of $K(F)$ and the equality

$$
\alpha_{F}^{-1}(H \Gamma)=\mathbf{K}(F)(H \times \Gamma) .
$$

Lemma 1.6 Let $S$ be an l-group that is countable at infinity and $\rho$ be an irreducible compact representation of $S$. Let $V \in \mathcal{M}(S)$. Then:

1. The map ev $: \rho \otimes \operatorname{Hom}_{S}(\rho, V) \rightarrow V$ is an embedding, and there exists a unique $S$-submodule $L \subset V$ such that $\operatorname{Im}(e v) \oplus L=V$.

2. If additionally a discrete group $\Gamma$ acts on $V$ commuting with the action of $S$, then $\operatorname{Hom}_{S}(\rho, V)$ can be embedded as a direct summand of $V$ as a $\Gamma$-module.

Proof 1. By [2, Theorem 7] we can decompose $V=\left(\oplus W_{i}\right) \oplus L$ with $W_{i} \cong \rho$ and $\rho \notin J H(L)$. This implies that $e v$ is an embedding and its image is $\bigoplus W_{i}$. This gives the existence of $L$. For its uniqueness, assume that we have a decomposition $\operatorname{Im}(\mathrm{ev}) \oplus L^{\prime}=$ $V$. This gives us a projection $p: V \rightarrow \operatorname{Im}(e v)$. Consider $\left.p\right|_{L}: L \rightarrow \operatorname{Im}(e v)$. We get $\left.p\right|_{L}=0$, which implies that $L \subset L^{\prime}$, and thus that $L=L^{\prime}$.

2. Note that the map $e v$ intertwines the action of $\Gamma$. By the previous item we can decompose $V \cong \rho \otimes \operatorname{Hom}_{S}(\rho, V) \oplus L$. The uniqueness of $L$ implies that it is $\Gamma$-invariant. Choosing a vector in $\rho$, we get an embedding of $\operatorname{Hom}_{S}(\rho, V)$ to $\rho \otimes \operatorname{Hom}_{S}(\rho, V)$ as a direct summand.

Proof of [1, Theorem 6.1]

Step 1: Analysis of $X$ as a $\Lambda_{\mathcal{Z}}$-space.

We define a filtration $\Lambda_{\mathcal{Z}}^{0} \subset \Lambda_{\mathcal{Z}}^{1} \subset \Lambda_{\mathcal{Z}}$ that will allow us to analyze the action of $\Lambda_{\mathcal{Z}}$ on $X$. By [1, Lemma 6.3.] we decompose $G^{1}=G^{0} \times \Lambda_{\mathcal{Z}}$. Let $\Lambda_{\mathcal{Z}}^{0}:=H \cap \Lambda_{\mathcal{Z}}$ and $\Lambda_{\mathcal{Z}}^{1}=H G^{0} \cap \Lambda_{\mathcal{Z}}$. Note that for any $x \in X$, we have $\Lambda_{\mathcal{Z}}^{1} \cdot x \subset G^{0} \cdot x$.

Up to replacing groups by their finite index subgroups, the filtration $\Lambda_{\mathcal{Z}}^{0} \subset \Lambda_{\mathcal{Z}}^{1} \subset$ $\Lambda_{\mathcal{Z}}$ splits. More precisely, denote

$$
\Gamma_{0}=\Lambda_{\mathcal{Z}}^{0} .
$$

We can find subgroups $\Gamma_{1}, \Gamma_{2} \subset \Lambda_{\mathcal{Z}}$ such that

$$
\Gamma_{0} \oplus \Gamma_{1}:=\Gamma_{0} \times \Gamma_{1} \subset \Lambda_{\mathcal{Z}}^{1}
$$

is of finite index and

$$
\Gamma_{0} \oplus \Gamma_{1} \oplus \Gamma_{2}:=\Gamma_{0} \times \Gamma_{1} \times \Gamma_{2} \subset \Lambda_{\mathcal{Z}}
$$

is of finite index.

Denote $G^{1, f}:=\left(\Gamma_{0} \times \Gamma_{1} \times \Gamma_{2}\right) G^{0} \subset G^{1}$. Note that $G^{1, f} \subset G$ is of finite index. 
Let $g_{1}, \ldots, g_{k} \in G$ be representatives of the cosets $G / G^{1, f}$ and let

$$
X^{0}:=G^{0} \cdot\left\{g_{1}, \ldots, g_{k}\right\} .
$$

Taking into account the fact that $G^{0}$ is normal in $G$, we get

$$
X \cong X^{0} \times \Gamma_{2}
$$

as $G^{1, f} \cong G^{0} \times \Gamma_{0} \times \Gamma_{1} \times \Gamma_{2}$-spaces. Here, the action of $G^{0} \times \Gamma_{1}$ is on the component $X^{0}$, the action of $\Gamma_{2}$ is on the component $\Gamma_{2}$, and the action of $\Gamma_{0}$ is trivial. Thus we have an isomorphism of $\Gamma_{0} \times \Gamma_{1} \times \Gamma_{2}$ representations:

$\mathcal{F}_{X, \mathcal{L}}((G, \rho))=\operatorname{Hom}_{G^{0}}(\rho, \mathcal{S}(X, \mathcal{L})) \cong \operatorname{Hom}_{G^{0}}\left(\rho, \mathcal{S}\left(X^{0},\left.\mathcal{L}\right|_{X^{0}}\right)\right) \otimes \mathbb{C}\left[\Gamma_{2}\right]$.

Here $\Gamma_{0}$ acts on the right-hand side by a character, $\Gamma_{1}$ acts on the component $\operatorname{Hom}_{G^{0}}\left(\rho, \mathcal{S}\left(X^{0},\left.\mathcal{L}\right|_{X^{0}}\right)\right)$, and $\Gamma_{2}$ acts on the component $\mathbb{C}\left[\Gamma_{2}\right]$.

Step 2: Proof that for any $G^{0} \times \Gamma_{1}$ equivariant line bundle $\mathcal{M}$ on $X^{0}$, the $\mathbb{C}\left[\Gamma_{1}\right]$-module $\mathcal{S}\left(X^{0}, \mathcal{M}\right)$ is free.

We will prove this using Corollary 1.3 and Lemma 1.5. It is sufficient to show that $\mathcal{S}\left(G^{0} g_{i},\left.\mathcal{M}\right|_{G^{0} g_{i}}\right)$ is a free $\mathbb{C}\left[\Gamma_{1}\right]$-module for any $1 \leq i \leq k$. Observe that $\Gamma_{1}$ acts freely on $G^{0} g_{i}$. Thus by Corollary 1.3 it is sufficient to show that $G^{0} g_{i} / \Gamma_{1}$ is Hausdorff. Furthermore, since $G^{0} g_{i} / \Gamma_{1} \subset X / \Gamma_{1}$ it is sufficient to show that the latter is Hausdorff. In other words, it must be shown that $H \Gamma_{1} \subset G$ is closed, which follows from Lemma 1.5.

Step 3: Proof that $\operatorname{Hom}_{G^{0}}\left(\rho, \mathcal{S}\left(X^{0},\left.\mathcal{L}\right|_{X^{0}}\right)\right)$ is a locally free $\mathbb{C}\left[\Gamma_{1}\right]$-module.

We will deduce this from the previous step using Lemma 1.6.

Since $\Gamma_{1}$ acts on $\rho$ by a character, we can twist the action of $\Gamma_{1}$ on $\left.\mathcal{L}\right|_{X^{0}}$ to get a $\left(G_{0}, \Gamma_{1}\right)$-equivariant line bundle $\mathcal{L}^{\prime}$ on $X^{0}$ such that

$$
\operatorname{Hom}_{G^{0}}\left(\rho, \mathcal{S}\left(X^{0},\left.\mathcal{L}\right|_{X^{0}}\right)\right) \cong \operatorname{Hom}_{G^{0}}\left(\rho, \mathcal{S}\left(X^{0}, \mathcal{L}^{\prime}\right)\right)
$$

as $\Gamma_{1}$-modules. Here, the action of $\Gamma_{1}$ on the RHS is on the values only. Let $\rho=$ $\rho_{1} \oplus \cdots \oplus \rho_{k}$ be the decomposition of $\rho$ to irreducible representations of $G_{0}$. It is enough to show that for all $i, \operatorname{Hom}_{G^{0}}\left(\rho_{i}, \mathcal{S}\left(X^{0}, \mathcal{L}^{\prime}\right)\right)$ is a locally free $\mathbb{C}\left[\Gamma_{1}\right]-$ module, when the action of $\mathbb{C}\left[\Gamma_{1}\right]$ is on the values only. By Lemma 1.6(2) the space $\operatorname{Hom}_{G^{0}}\left(\rho_{i}, \mathcal{S}\left(X^{0}, \mathcal{L}^{\prime}\right)\right.$ can be embedded as a direct summand of $\mathcal{S}\left(X^{0}, \mathcal{L}^{\prime}\right)$. The latter is free (as shown above), hence $\operatorname{Hom}_{G^{0}}\left(\rho_{i}, \mathcal{S}\left(X^{0}, \mathcal{L}^{\prime}\right)\right)$ is a locally free $\mathbb{C}\left[\Gamma_{1}\right]$-module.

Step 4: The end of the proof.

We will deduce the theorem from the previous step using [1, Lemma 6.2.].

Let $L:=\operatorname{Spec}\left(\mathbb{C}\left[\Gamma_{0} \times \Gamma_{1} \times \Gamma_{2}\right]\right)$. The map $\pi: \mathfrak{X}_{G}=\operatorname{Spec}\left(\mathbb{C}\left[G / G^{0}\right]\right) \rightarrow L$ is a finite étale morphism. Using Equation (1) together with the previous step, we conclude that $\pi_{*}\left(\mathcal{F}_{X, \mathcal{L}}((G, \rho))\right)$ is the direct image of a free sheaf on a coset of $\operatorname{Spec}\left(\mathbb{C}\left[\Gamma_{1} \times \Gamma_{2}\right]\right)$.

Therefore [1, Lemma 6.2.] implies the assertion of the Theorem.

\section{Correction of [1, Appendix B]}

[1, Lemma B.2] is wrong as stated. However it holds true under the further assumption that either $K$ is flat or $N$ is projective. Corollary [1, Corollary B.4] have to be changed accrodingly. The rest of the Appendix holds without changes. 
In the following we provide a corrected version of the lemma, its proof and a discussion of its use in [1, Appendix B].

Lemma 2.1 Let $A$ and $B$ be associative unital Noetherian algebras. Let $N$ be a finitely generated left $A$-module, $K$ be a left $B$-module, and $M$ be an $(A, B)$-bi-module. Let

$$
b: \operatorname{Hom}_{A}(N, M) \times K \rightarrow \operatorname{Hom}_{A}\left(N, M \otimes_{B} K\right)
$$

be the B-bilinear map given by

$$
b(\phi, k)(n)=\phi(n) \otimes k .
$$

Let

$$
\beta: \operatorname{Hom}_{A}(N, M) \otimes_{B} K \rightarrow \operatorname{Hom}_{A}\left(N, M \otimes_{B} K\right)
$$

be the corresponding morphism.

(1) if $K$ is a flat $B$-module, then $\beta$ is an isomorphism.

(2) if $N$ is a projective A-module, then $\beta$ is an isomorphism.

Proof We first consider the case where $K$ is flat. Since $N$ is finitely generated and $A$ is Noetherian we can find a finite presentation

$$
A^{n} \rightarrow A^{m} \rightarrow N \rightarrow 0
$$

of $N$. Applying the fuctor $\operatorname{Hom}_{A}(\bullet, M)$ to (2), we obtain the following exact sequences:

$$
0 \rightarrow \operatorname{Hom}_{A}(N, M) \rightarrow M^{m} \rightarrow M^{n} .
$$

Since $K$ is flat we get an exact sequence

$$
0 \rightarrow \operatorname{Hom}_{A}(N, M) \otimes_{B} K \rightarrow M^{m} \otimes_{B} K \rightarrow M^{n} \otimes_{B} K .
$$

Applying the functor $\operatorname{Hom}_{A}\left(\bullet, M \otimes_{B} K\right)$ to (2), we also get

$$
0 \rightarrow \operatorname{Hom}_{A}\left(N, M \otimes_{B} K\right) \rightarrow\left(M \otimes_{B} K\right)^{m} \rightarrow\left(M \otimes_{B} K\right)^{n} .
$$

Equivalenty,

$$
0 \rightarrow \operatorname{Hom}_{A}\left(N, M \otimes_{B} K\right) \rightarrow M^{m} \otimes_{B} K \rightarrow M^{n} \otimes_{B} K
$$

is exact. Consider now the diagram

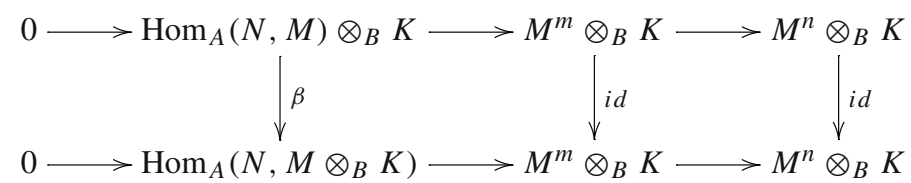

Applying the 5-Lemma we deduce that $\beta$ is an isomorphism as claimed.

To prove the second item, we use the projectivity and finite generation of $N$ to write $A^{n}=N \bigoplus N^{\prime}$, for some integer $n$ and an $A$-module $N^{\prime}$. Define

$$
\beta^{\prime}: \operatorname{Hom}_{A}\left(N^{\prime}, M\right) \otimes_{B} K \rightarrow \operatorname{Hom}_{A}\left(N^{\prime}, M \otimes_{B} K\right)
$$

in a similar fashion to $\beta$. It is easy to see that $\beta \oplus \beta^{\prime}: M^{n} \otimes_{B} K \rightarrow\left(M \otimes_{B} K\right)^{n}$ is an isomorphsm. This imply that $\beta$ is an isomorphism. 


\subsection{The usage of [1, Lemma B.2] in [1, Appendix B]}

[1, Lemma B.2] is used in two places in [1]: [1, Lemma B.5] and [1, Corollary B.4].

In [1, Lemma B.5] we use [1, Lemma B.2] for the case were $B$ is $\mathcal{H}\left(G^{0}, K\right)$ and $K$ is $\mathcal{H}(G, K)$. It is easy to see that the module $K$ is a free $B$-module and hence it is flat. Thus, this case is covered by the first item of Lemma 2.1.

For the validity of [1, Corollary B.4] we have to add the assumption that $V$ is projective. With this further assumption [1, Corollary B.4] is true and is indeed a consequence of the second item of Lemma 2.1 (that is, the corrected version of [1, Lemma B.2] provided above).

In [1, Appendix B] we use [1, Corollary B.4] only in Proposition [1, Lemma B.6]. There we apply [1, Corollary B.4] with $V=i n d_{M^{0}}^{M}\left(\left.\rho\right|_{M^{0}}\right)$ where $\rho$ is a cuspidal representation of $M$ and $M^{0}<M$ the subgroup generated by compact subgroups of $M$. In this case, by a result of Harish-Chandra, the module $V$ is indeed projective and the conclusion is valid.

Acknowledgements We would like to thank Raphaël Beuzart-Plessis for detecting the error in [1, §6] and Yangyu Fan for detecting the error in [1, Lemma B.2].

\section{References}

1. Aizenbud, A., Sayag, E.: Homological multiplicities in representation theory of $p$-adic groups. Math. Z. 294, 451-469 (2020)

2. Bernstein, J.N., Rumelhart, K.: Lectures on $p$-adic groups. http://www.math.tau.ac.il/ bernstei/ Publication_list/publication_texts/Bernst_Lecture_p-adic_repr.pdf

3. Bernstein, J., Zelevinsky, A.: Representations of $G L(n, F)$ where $F$ is a non-Archimedean local field. Russ. Math. Surv. 31(3), 1-68 (1976)

4. Sakellaridis, Y., Venkatesh, A.: Periods and harmonic analysis on spherical varieties. Astérisque (396), 360 (2017)

Publisher's Note Springer Nature remains neutral with regard to jurisdictional claims in published maps and institutional affiliations. 